\title{
Modified Gauss-Bonnet gravity with Lagrange multiplier constraint as mimetic theory
}

\author{
Artyom V. Astashenok ${ }^{1}$, Sergei D. Odintsov ${ }^{2,3}$, V.K. Oikonomou ${ }^{4,5}$ \\ 1 Baltic Federal University of I. Kant, Department of Theoretical Physics, 236041, 14, Nevsky st., Kaliningrad, Russia \\ ${ }^{2}$ Instituciò Catalana de Recerca i Estudis Avançats (ICREA), Barcelona, Spain \\ ${ }^{3}$ Institut de Ciencies de l'Espai (CSIC-IEEC), Campus UAB, Campus UAB, \\ Carrer de Can Magrans, s/n 08193 Cerdanyola del Valles, Barcelona, Spain \\ 4) Department of Theoretical Physics, Aristotle University of Thessaloniki, 54124 Thessaloniki, Greece \\ 5) National Research Tomsk State University, 634050 Tomsk and Tomsk State Pedagogical University, 634061 Tomsk, Russia
}

\begin{abstract}
In this paper we propose and extensively study mimetic $f(\mathcal{G})$ modified gravity models, with various scenarios of cosmological evolution, with or without extra matter fluids. The easiest formulation is based on the use of Lagrange multiplier constraint. In certain versions of this theory, it is possible to realize accelerated expansion of the Universe or even unified evolution which includes inflation with dark energy, and at the same time in the same theoretical framework, dark matter is described by the theory. This is achieved by the re-parametrization of the metric tensor, which introduces a new degree of freedom in the cosmological equations and leads to appearance of the mimetic "dark matter" component. In the context of mimetic $f(\mathcal{G})$ theory, we also provide some quite general reconstruction schemes, which enable us to find which $f(\mathcal{G})$ gravity generates a specific cosmological evolution. In addition, we also provide the general reconstruction technique for Lagrange multiplier $f(\mathcal{G})$ gravity. All our results are accompanied by illustrative examples, with special emphasis on bouncing cosmologies.
\end{abstract}

\section{INTRODUCTION}

The recent observational data [1, 2], have increased the scientific interest in cosmology, since early-time acceleration is favored by present observations. In addition to this early-time acceleration, in the late 90's, the late-time acceleration (including that from modified gravity [3, [4]) was also verified by the observations [5]. Since then a great task for cosmologists is to consistently describe these two acceleration eras, and if possible, to include them in the same theoretical framework as it was first proposed in [7]. Modified gravity [ 6], is one appealing candidate for a complete cosmological theory, in the context of which late-time acceleration can be consistently described but more importantly, the unification of early-time acceleration with late-time acceleration [7, 8] can be achieved (for a general review, see 6]). There are many types of modified theories of gravity with higher-derivative terms, such as $f(R)$ modified gravity 9], $f(\mathcal{G})$ theories of gravity [23], etc. In this paper, we shall introduce a new type of mimetic $f(\mathcal{G})$ theory of gravity, which as we shall demonstrate, can successfully describe a variety of cosmological scenarios. It is inspired by mimetic dark matter models $10-12$ and serves as a generalization of both $f(\mathcal{G})$ and mimetic dark matter models.

The mimetic dark matter models [10 12], are generalizations of general relativity, at which general relativity is modified in a minimal way, so that the conformal symmetry not to be violated. In the theoretical formalism of mimetic dark matter theories, an extra internal degree of freedom appears, which can describe dark matter via such mimetic gravity theory [10] (see also Refs. 11, 12]). In order for the conformal symmetry not to be violated, the metric is not regarded as the fundamental variable of this gravitational theory, but is decomposed into a new metric and a scalar field, with the latter being the extra gravitational degree of freedom which actually describes dark matter.

A recent study in which the formalism of mimetic gravity was used in the context of $f(R)$ gravity was done in Ref. [13]. As was shown in that work, in the context of mimetic $f(R)$ gravity and specifically of modified $f(R)$ gravity with Lagrange multiplier constraint, it is possible to realize the unification of early-time and late-time acceleration eras as well as having Dark Matter in unified scenario.

It is of great importance to understand how universal is such an approach to the unification of the three dark sectors of the Universe.

In this paper we consider modified Gauss-Bonnet $f(\mathcal{G})$ gravity, which was introduced as gravitational alternative to dark energy in Ref. 14], but generalized in such a way that the Lagrangian multiplier constraint is used, as done in Ref. [15]. The specific choice of the Lagrange multiplier constraint, renders such a theory as a mimetic modified Gauss-Bonnet gravity of certain type. The accelerating cosmology generated by such a mimetic gravity, is studied in detail and we also investigate which $f(\mathcal{G})$ gravity can realize various cosmological scenarios, with special emphasis to bouncing cosmologies [16 21]. We need to note that the idea of obtaining a bouncing solution in the frame of Lagrange multiplier modified gravity was studied for the first time in 22], which is very relevant in spirit to our approach. In addition, we also introduce the mimetic gravity formalism, in the context of a general Jordan frame $f(\mathcal{G})$ gravity. As a general remark, we must note that within the context of mimetic $f(\mathcal{G})$ gravity and Lagrange multiplier modified $f(\mathcal{G})$ gravity, it is possible that there exists a large number of $f(\mathcal{G})$ theories which can consistently describe a given cosmological evolution. 
This paper is organized as follows: In section II we present the theoretical formalism of mimetic Gauss-Bonnet gravity. The most important argument is based on the fact that the metric is redefined, and all cosmological equations are obtained by varying the redefined action with respect to redefined metric $\hat{g}_{\mu \nu}=-\hat{g}^{\rho \sigma} \partial_{\rho} \phi \partial_{\sigma} \phi \hat{g}_{\mu \nu}$ and mimetic scalar field $\phi$ instead of $g_{\mu \nu}$. In section III we investigate how various cosmological scenarios can be realized in the context of mimetic $f(\mathcal{G})$ gravity, with special emphasis to cosmological bounces. In section IV, we consider a generalization of $f(\mathcal{G})$ theory, based on the introduction of a Lagrange multiplier, which includes the mimetic case. Several examples are worked out and also comparison to the standard Einstein-Hilbert gravity with Lagrange multiplier, worked out in [11] is performed. Moreover, we also compare the resulting modified $f(\mathcal{G})$ gravity, mimetic or Lagrange multiplier modified, with the standard $f(\mathcal{G})$ gravity picture. The conclusions follow in the end of the paper.

\section{MIMETIC $f(\mathcal{G})$ GRAVITY}

Consider a general $f(\mathcal{G})$ gravity, with the Jordan frame action being equal to,

$$
S=\int d^{4} x \sqrt{-g}\left[\frac{R}{2 \kappa^{2}}+f(\mathcal{G})\right]+S_{m}
$$

where $S_{m}$ stands for the action of matter-fluids, which induces the energy momentum tensor $T_{\mu \nu}$ in the field equations (for some incomplete list of works on cosmology of such theory, see [23]). We adopt the standard geometrical conventions of Einstein-Hilbert gravity, with regards to the commutative connection of the underlying Riemannian spacetime, $R$ is the Ricci scalar, and the function $f(\mathcal{G})$ corresponds to a generic globally differentiable function of the Gauss-Bonnet topological invariant $\mathcal{G}$, which is equal to,

$$
\mathcal{G}=R^{2}-4 R_{\mu \nu} R^{\mu \nu}+R_{\mu \nu \lambda \sigma} R^{\mu \nu \lambda \sigma}
$$

where $R_{\mu \nu}$ and $R_{\mu \nu \lambda \sigma}$ are the Ricci and Riemann tensors, respectively. We adopted the signature for the Riemannian metric as $(-+++)$ and also we assume that $\kappa^{2}=8 \pi G / c^{4}=1$, where $G$ is the standard Newtonian gravitational coupling.

In order to obtain the mimetic $f(\mathcal{G})$, the following parametrization of metric is assumed [10 12]:

$$
g_{\mu \nu}=-\hat{g}^{\rho \sigma} \partial_{\rho} \phi \partial_{\sigma} \phi \hat{g}_{\mu \nu}
$$

By varying the metric we obtain the following relation,

$$
\begin{gathered}
\delta g_{\mu \nu}=\hat{g}^{\rho \tau} \delta \hat{g}_{\tau \omega} \hat{g}^{\omega \sigma} \partial_{\rho} \phi \partial_{\sigma} \phi \hat{g}_{\mu \nu}-\hat{g}^{\rho \sigma} \partial_{\rho} \phi \partial_{\sigma} \phi \delta \hat{g}_{\mu \nu}- \\
-2 \hat{g}^{\rho \sigma} \partial_{\rho} \phi \partial_{\sigma} \delta \phi \hat{g}_{\mu \nu} .
\end{gathered}
$$

In addition, by varying the action (10), with respect to the redefined metric $\hat{g}_{\mu \nu}$, instead of the standard Jordan frame metric $g_{\mu \nu}$, and with respect to the mimetic scalar $\phi$, the resulting field equations take the following form,

$$
\begin{gathered}
R_{\mu \nu}-\frac{1}{2} R g_{\mu \nu}+ \\
+8\left[R_{\mu \rho \nu \sigma}+R_{\rho \nu} g_{\sigma \mu}-R_{\rho \sigma} g_{\nu \mu}-R_{\mu \nu} g_{\sigma \rho}+R_{\mu \sigma} g_{\nu \rho}+\frac{R}{2}\left(g_{\mu \nu} g_{\sigma \rho}-g_{\mu \sigma} g_{\nu \rho}\right)\right] \nabla^{\rho} \nabla^{\sigma} f_{\mathcal{G}}+\left(f_{\mathcal{G}} \mathcal{G}-f(\mathcal{G})\right) g_{\mu \nu}+ \\
+\partial_{\mu} \phi \partial_{\nu} \phi\left(-R+8\left(-R_{\rho \sigma}+\frac{1}{2} R g_{\rho \sigma}\right) \nabla^{\rho} \nabla^{\sigma} f_{\mathcal{G}}+4\left(f_{\mathcal{G}} \mathcal{G}-f(\mathcal{G})\right)\right)= \\
=T_{\mu \nu}+\partial_{\mu} \phi \partial_{\nu} \phi T
\end{gathered}
$$

where $f_{\mathcal{G}}$ stands for $f_{\mathcal{G}}=d f(\mathcal{G}) / d \mathcal{G}$. We also note that the covariant derivative $\nabla_{\mu}$, acts on vectors as $\nabla_{\mu} V_{\nu}=$ $\partial_{\mu} V_{\nu}-\Gamma_{\mu \nu}^{\lambda} V_{\lambda}$ and accordingly it acts on the metric $g_{\mu \nu}$.

By varying the action (10) with respect to the scalar field $\phi$, we obtain the following equation,

$$
\nabla^{\mu}\left(\partial_{\mu} \phi\left(-R+8\left(-R_{\rho \sigma}+\frac{1}{2} R g_{\rho \sigma}\right) \nabla^{\rho} \nabla^{\sigma} f_{\mathcal{G}}+4\left(f_{\mathcal{G}} \mathcal{G}-f(\mathcal{G})\right)-T\right)\right)=0
$$


Assuming a spatially-flat Friedmann-Robertson-Walker (FRW) metric, with metric

$$
d s^{2}=-d t^{2}+a^{2}(t)\left(d x^{2}+d y^{2}+d z^{2}\right)
$$

the scalar curvature and the Gauss-Bonnet invariant have the following form:

$$
\begin{aligned}
& R=6\left(\frac{\ddot{a}}{a}+\frac{\dot{a}^{2}}{a^{2}}\right)=6\left(\dot{H}+2 H^{2}\right), \\
& \mathcal{G}=-24 \frac{\ddot{a} \dot{a}^{2}}{a^{3}}=-24 H^{2}\left(\dot{H}+H^{2}\right) .
\end{aligned}
$$

where $H(t)$ denotes as usual the Hubble rate $H(t)=\dot{a}(t) / a(t)$. From Eq. (3) we find that

$$
g^{\mu \nu} \partial_{\mu} \phi \partial_{\nu} \phi=-1
$$

and since the scalar field $\phi$ depends only on the cosmic time, this results to the constraint $\phi=t$. Taking into account the aforementioned constraint, we can obtain the $(t, t)$ component of the expression given in (4):

$$
2 \dot{H}+3 H^{2}+16 H\left(\dot{H}+H^{2}\right) \frac{d f_{\mathcal{G}}}{d t}+8 H^{2} \frac{d^{2} f_{\mathcal{G}}}{d t^{2}}-\left(f_{\mathcal{G}} \mathcal{G}-f(\mathcal{G})\right)=-p .
$$

The same equation results if we consider the $(r, r)$ component. By integrating Eq. (5), we obtain the following relation,

$$
-R+8\left(-R_{\rho \sigma}+\frac{1}{2} R g_{\rho \sigma}\right) \nabla^{\rho} \nabla^{\sigma} f_{\mathcal{G}}+4\left(f_{\mathcal{G}} \mathcal{G}-f(\mathcal{G})\right)+\rho-3 p=-\frac{C}{a^{3}}
$$

and finally this results to,

$$
\dot{H}+2 H^{2}+4 H^{2} \frac{d^{2} f_{\mathcal{G}}}{d t^{2}}+4 H\left(2 \dot{H}+3 H^{2}\right) \frac{d f_{\mathcal{G}}}{d t}+\frac{2}{3}\left(f_{\mathcal{G}} \mathcal{G}-f(\mathcal{G})\right)+\frac{\rho}{6}-\frac{p}{2}=-\frac{C}{a^{3}} .
$$

where $C$ is an arbitrary constant.

Combining Eqs. (8) and (10), we obtain:

$$
\dot{H}+4 H^{2} \frac{d^{2} f_{\mathcal{G}}}{d t^{2}}+4 H\left(2 \dot{H}-H^{2}\right) \frac{d f_{\mathcal{G}}}{d t}=-\frac{1}{2}(\rho+p)-\frac{C}{a^{3}} .
$$

where for simplicity we redefined the constant $C$. It is convenient to introduce the function $g(t)$, which is defined to be equal to,

$$
g(t)=\frac{d f_{\mathcal{G}}}{d t}
$$

which satisfies the equation:

$$
4 H^{2} \frac{d g(t)}{d t}+4 H\left(2 \dot{H}-H^{2}\right) g(t)=-\dot{H}-\frac{1}{2}(\rho+p)-\frac{C}{a^{3}} .
$$

For $C=0$ Eqs. (8) and (11) coincide with the Friedmann equations in ordinary $f(\mathcal{G})$ gravity. If the right hand side of Eq. (12 is zero, that is, $B(t)=-\dot{H}-\frac{1}{2}(\rho+p)-\frac{C}{a^{3}}=0$ then the solution to this equation can be written as follows,

$$
g(t)=g_{0}\left(\frac{H_{0}}{H}\right)^{2} \exp \left(\int_{0}^{t} H d t\right)
$$

In the above equation, $g_{0}$ is a constant and $H_{0}=H(0)$. In the case that the right hand of Eq. (12) is non-zero, that is when $B(t) \neq 0$, the solution $g(t)$ reads,

$$
g(t)=g_{0}\left(\frac{H_{0}}{H}\right)^{2} \exp \left(\int_{0}^{t} H d t\right)+\frac{1}{4 H^{2}} \int_{0}^{t} d t_{1} B\left(t_{1}\right) \exp \left(\int_{t_{1}}^{t} H(\tau) d \tau\right) .
$$


Therefore one can specify the cosmic evolution of the Universe, in terms of the Hubble rate $H(t)$ and then obtain the function $g(t)$. Then by integrating this, with respect to the cosmic time, we can obtain the function $f(t)$, that is,

$$
f_{\mathcal{G}}(t)=\int g(t) d t
$$

Using the explicit dependence of the Gauss-Bonnet invariant on the cosmic time $t$, one can get the explicit form of the function $t(\mathcal{G})$ and therefore find the function $f_{\mathcal{G}}(\mathcal{G})$. Then, upon integrating with respect to $\mathcal{G}$, we easily obtain the mimetic $f(\mathcal{G})$ gravity. In the next section, we shall present some illustrative examples, in order to demonstrate explicitly how the reconstruction method works, and also in order to see the differences between Jordan frame mimetic $f(\mathcal{G})$ gravity and ordinary Jordan frame $f(\mathcal{G})$ gravity.

\section{COSMOLOGICAL MODELS WITH VARIOUS EVOLUTIONS FROM MIMETIC $f(\mathcal{G})$}

\section{A. A general example}

We start off with the cosmological evolution of the Universe, with a Hubble rate equal to,

$$
H(t)=\frac{H_{0}}{1+\alpha t}, \quad \alpha>0
$$

which could correspond for example to early-time acceleration from the moment $t=0$ to some later time $t$, with $t>>\alpha^{-1}$. The corresponding scale factor for the Hubble parameter (III A) is equal to,

$$
a(t)=a_{0}(1+\alpha t)^{H_{0} / \alpha}
$$

The corresponding Gauss-Bonnet invariant is,

$$
\mathcal{G}=24 \frac{H_{0}^{2}\left(\alpha-H_{0}\right)}{(1+\alpha t)^{3}} .
$$

The solution of Eq. (12) with zero right hand side $(B=0)$, is equal to,

$$
g(t)=g_{0}(1+\alpha t)^{H_{0} / \alpha+2} .
$$

For an empty universe without matter and mimetic dark matter $(\rho=p=C=0)$ the function $g(t)$ is equal to,

$$
g(t)=g_{0}(1+\alpha t)^{H_{0} / \alpha+2}-\frac{1}{4 H_{0}} \frac{1+\alpha t}{\alpha+H_{0}} .
$$

Then, the resulting expression for the function $f_{\mathcal{G}}(t)$ is equal to,

$$
f_{\mathcal{G}}(t)=f_{0}(1+\alpha t)^{H_{0} / \alpha+3}-\frac{1}{8 \alpha H_{0}} \frac{(1+\alpha t)^{2}}{\alpha+H_{0}} .
$$

Using the Gauss-Bonnet invariant (IIIA), we can express the function $f_{\mathcal{G}}(t)$, as a function of the Gauss-Bonnet invariant $\mathcal{G}$,

$$
\begin{gathered}
f_{\mathcal{G}}=A \mathcal{G}^{-1-H_{0} / 3 \alpha}+D \mathcal{G}^{-2 / 3} \\
D=-\frac{1}{8 \alpha H_{0}\left(\alpha+H_{0}\right)}\left(24 H_{0}^{2}\left(\alpha-H_{0}\right)\right)^{2 / 3} .
\end{gathered}
$$

Finally, integrating the above equation, with respect to the Gauss-Bonnet invariant $\mathcal{G}$, we obtain the resulting nonmimetic $f(\mathcal{G})$ gravity,

$$
f(\mathcal{G})=F_{0} \mathcal{G}^{-H_{0} / \alpha}+3 D \mathcal{G}^{1 / 3}
$$


For a Universe filled with matter and mimetic dark matter, which means that $C \neq 0, \rho=\rho_{0} a^{-3}, p=0$, the corresponding solution $g(t)$ has the following form,

$$
g(t)=g_{0}(1+\alpha t)^{H_{0} / \alpha+2}-\frac{1}{4 H_{0}} \frac{1+\alpha t}{\alpha+H_{0}}+\frac{\tilde{C}}{4 H_{0}-\alpha} \frac{1}{4 H_{0}^{2}}(1+\alpha t)^{3\left(1-H_{0} / \alpha\right)}, \quad \tilde{C}=C+\rho_{0},
$$

and the corresponding solution $f_{\mathcal{G}}(t)$ reads,

$$
f_{\mathcal{G}}(t)=A \mathcal{G}^{-1-H_{0} / 3 \alpha}+D \mathcal{G}^{-2 / 3}+E \mathcal{G}^{-4 / 3+H_{0} / \alpha}
$$

where $E$ stands for,

$$
E=\frac{\tilde{C}}{4 H_{0}^{2}} \frac{\alpha}{\left(4 H_{0}-\alpha\right)\left(4 \alpha-H_{0}\right)}\left(24 H_{0}^{2}\left(\alpha-H_{0}\right)\right)^{4 / 3-H_{0} / \alpha},
$$

The resulting mimetic $f(\mathcal{G})$ gravity easily follows,

$$
f(\mathcal{G})=F_{0} \mathcal{G}^{-H_{0} / \alpha}+3 D \mathcal{G}^{1 / 3}+\frac{3 \alpha}{3 H_{0}-\alpha} E \mathcal{G}^{-1 / 3+H_{0} / \alpha} .
$$

By looking Eqs. (19) and (16), we can see the difference between non-mimetic and mimetic $f(\mathcal{G})$ gravity. Notice that in the mimetic gravity case, a given cosmological evolution can be realized by a family of $f(\mathcal{G})$ functions. For example by putting $C=-\rho_{0}$ and therefore the last term can be omitted in Eq. (17). It is worthy to note that in ordinary $f(\mathcal{G})$ gravity with matter fluids, we have no such freedom in choosing the $f(\mathcal{G})$ function, and a unique solution for $f(\mathcal{G})$ exists (in (17) one need to change $\tilde{C} \rightarrow \rho_{0}$ ).

Let us study another case, in which the cosmological evolution is described by the following Hubble rate,

$$
H=\frac{H_{0}}{1+\beta^{2} t^{2}}
$$

We consider the simple case for which $g_{0}=0$ and $\rho=p=C=0$, and the solution $g(t)$ is equal to,

$$
g(t)=-\frac{\beta^{2}\left(1+\beta^{2} t^{2}\right)\left(1+H_{0} t-\beta^{2} t^{2}\right)}{2 H_{0}\left(H_{0}^{2}+4 \beta^{2}\right)}
$$

The Gauss-Bonnet invariant is,

$$
\mathcal{G}=\frac{24 H_{0}^{2}\left(2 \beta^{2} t-H_{0}\right)}{\left(\beta^{2} t^{2}+1\right)^{3}}
$$

For $t \rightarrow \infty$ we have the following asymptotic behavior:

$$
\begin{gathered}
g(t) \approx \frac{\beta^{6} t^{4}}{2 H_{0}\left(H_{0}^{2}+4 \beta^{2}\right)} \\
\mathcal{G} \approx \frac{48 H_{0}^{2}}{\beta^{4} t^{5}}
\end{gathered}
$$

Therefore, the function $f_{\mathcal{G}}(\mathcal{G})$ is equal to,

$$
f_{\mathcal{G}}(\mathcal{G}) \approx \frac{\beta^{14 / 5}}{2\left(48 H_{0}\right)^{-4 / 5} H_{0}\left(H_{0}^{2}+4 \beta^{2}\right)} \mathcal{G}^{-4 / 5}
$$

and upon integrating with respect to $\mathcal{G}$, we finally obtain the $f(\mathcal{G})$ gravity,

$$
f(\mathcal{G}) \approx \frac{5 \beta^{14 / 5}}{2\left(48 H_{0}\right)^{-4 / 5} H_{0}\left(H_{0}^{2}+4 \beta^{2}\right)} \mathcal{G}^{1 / 5} .
$$




\section{B. Reconstruction of mimetic $f(\mathcal{G})$ models from bounce cosmological evolution}

Using the method we described in the previous sections, it is possible to reconstruct an $f(\mathcal{G})$ model for given cosmological evolution with scale factor $a(t)$. In this section we shall consider bouncing cosmologies, and we investigate which $f(\mathcal{G})$ model can generate such a cosmology, in the presence of matter and mimetic dark matter.

\section{Superbounce from mimetic $f(\mathcal{G})$ gravity}

In the context of $f(\mathcal{G})$ modified gravity, various bouncing Universe scenarios can be materialized straightforwardly, which in the context of Einstein-Hilbert general relativity could only be realized if the null energy condition is violated for a period of time. For reviews and an important stream of papers on bouncing cosmology see [16 20] and references therein. A bounce evolution has the following characteristics: The Universe starts with a contraction up to a point where a non-zero minimal radius is reached, where the scale factor is non-vanishing too, and then the Universe starts to expand again. In this way the singularity problem of the scale factor, which is known as the initial singularity, is resolved in a self-consistent way. Therefore, bouncing cosmology serves as a convenient way to avoid singularities of crushing type, where the geodesics incompleteness occurs. Let the bounce occur at some time $t_{s}$, then for $t=t_{s}$ the Hubble rate is zero, that is $H\left(t_{s}\right)=0$, while during the expanding phase, $H(t)>0$, and during the contracting phase, $H(t)<0$.

Therefore the bouncing cosmology is one appealing candidate for the consistent description of the Universe's evolution. Within this context, we shall make use of two bouncing cosmologies for our reconstruction examples.

We start of with the superbounce model [21], which has the following scale factor,

$$
a(t)=\left(-t+t_{s}\right)^{\frac{2}{c^{2}}}
$$

while the corresponding Hubble rate is,

$$
H(t)=-\frac{2}{c^{2}\left(-t+t_{s}\right)}
$$

Using Eqs. (22) and (23) and substituting to Eq. (14), the solution $g(t)$ easily follows,

$$
g(t)=\frac{1}{4} c^{4} g_{0} H_{0}^{2}\left(t-t_{s}\right)^{\frac{2}{c^{2}}}\left(-t+t_{s}\right)^{2}-\frac{1}{32} c^{6}\left(t-t_{s}\right)^{\frac{2}{c^{2}}}\left(-t+t_{s}\right)^{2}\left(\frac{2}{-2+c^{2}}+\frac{c^{2} C\left(-t+t_{s}\right)^{2-\frac{6}{c^{2}}}}{8-6 c^{2}+c^{4}}+\frac{c^{2}\left(t-t_{s}\right)^{2}(p+\rho)}{2\left(2+3 c^{2}+c^{4}\right)}\right)
$$

The Gauss-Bonnet invariant for the Hubble rate of Eq. (23) is equal to,

$$
\mathcal{G}=-96 c^{2} t^{2}\left(2 c+4 c^{2} t^{2}\right)
$$

and by solving Eq. (25) with respect to $t$ we obtain two real solutions,

$$
t_{1}=\frac{-2 \sqrt{2} 3^{1 / 4} c^{4} \sqrt{G}\left(c^{8}\left(-2+c^{2}\right) G\right)^{1 / 4}+c^{8} G t_{s}}{c^{8} G}, \quad t_{2}=\frac{2 \sqrt{2} 3^{1 / 4} c^{4} \sqrt{G}\left(c^{8}\left(-2+c^{2}\right) G\right)^{1 / 4}+c^{8} G t_{s}}{c^{8} G}
$$

By substituting Eq. (26) to Eq. (24) and integrating over $\mathcal{G}$, we obtain two different functional forms of mimetic $f(\mathcal{G})$ gravity, which are of the following form,

$$
\begin{aligned}
& f(\mathcal{G})=-12\left(-2+c^{2}\right)\left(t(\mathcal{G})-t_{s}\right)^{-1+\frac{2}{c^{2}}}\left(\frac{4\left(8 g_{0} H_{0}^{2}+c^{2}\left(1-4 g_{0} H_{0}^{2}\right)\right)}{\left(-2+c^{2}\right)^{2}\left(2+3 c^{2}\right)}\right. \\
& \left.-\frac{2 c^{4} C\left(-t(\mathcal{G})+t_{s}\right)^{2-\frac{6}{c^{2}}}}{\left(-4+c^{2}\right)^{2}\left(8-14 c^{2}+5 c^{4}\right)}-\frac{c^{4}\left(t(\mathcal{G})-t_{s}\right)^{2}(p+\rho)}{\left(2+c^{2}\right)^{2}\left(2+7 c^{2}+5 c^{4}\right)}\right)
\end{aligned}
$$

where $t(\mathcal{G})=t_{1,2}$ with $t_{1}, t_{2}$ being defined in Eq. (26).

Let us here compare the Jordan frame mimetic $f(\mathcal{G})$ modified gravity solutions we found, that generate the superbounce (22), with the solutions of non-mimetic $f(\mathcal{G})$ gravity in the Jordan frame. The non-mimetic $f(\mathcal{G})$ gravity solutions that generate the superbounce (22) are the following 21]:

$$
f(\mathcal{G})=\frac{t_{*}^{2} \mathcal{G}-4 \sqrt{(-11+a) a^{3} \mathcal{G}}}{4 a+4 a^{2}}, \quad f(\mathcal{G})=\frac{t_{*}^{2} \mathcal{G}-2 \sqrt{(-11+a) a^{3} \mathcal{G}}(1+\mathcal{G})}{4 a(1+a) \mathcal{G}}
$$


where $a=2 / c^{2}$. Comparing Eqs. (27) and (28), we can see the obvious functional differences. But there is also another important difference, having to do with the freedom in choosing the solutions in the case of mimetic $f(\mathcal{G})$ gravity, an issue we already discussed earlier. Notice also that there is a similarity between the mimetic and nonmimetic $f(\mathcal{G})$ gravities, which is that in both cases, the resulting $f(\mathcal{G})$ gravity consists of two distinct solutions, when the superbounce cosmological evolution is taken into account.

\section{Generic bounce scenario from mimetic $f(\mathcal{G})$ gravity}

Another interesting bouncing cosmology scenario is described by the following scale factor,

$$
a(t)=e^{c t^{2}}
$$

with $c$ some arbitrary constant and the scale factor is normalized again so that at the bouncing point, which is $t=0$, the scale factor is equal to $a(0)=1$. The corresponding Hubble rate is particularly simple, and it is equal to,

$$
H(t)=2 c t
$$

Using the Hubble rate (30) and by substituting to Eq. (14), we get the function $g(t)$,

$$
\begin{aligned}
& g(t)=\frac{C e^{-2 c t^{2}}}{64 c^{3} t}-\frac{e^{c t^{2}}}{16 c^{2} t}-\frac{e^{c t^{2}} g_{0} H_{0}^{2}}{4 c^{2} t}-\frac{e^{c t^{2}} p}{64 c^{3} t}-\frac{e^{c t^{2}} \rho}{64 c^{3} t}+\frac{C \sqrt{\frac{\pi}{2}} \operatorname{erf}[\sqrt{2} \sqrt{c t}]}{32 c^{5 / 2}}+\frac{\sqrt{\pi} \operatorname{erfi}(\sqrt{c} t)}{16 c^{3 / 2}} \\
& +\frac{g_{0} H_{0}^{2} \sqrt{\pi} \operatorname{erfi}(\sqrt{c} t)}{4 c^{3 / 2}}+\frac{p \sqrt{\pi} \operatorname{erfi}(\sqrt{c} t)}{64 c^{5 / 2}}+\frac{\sqrt{\pi} \rho \operatorname{erfi}(\sqrt{c} t)}{64 c^{5 / 2}} \\
& -\frac{C t_{2} F_{2}\left(\frac{1}{2} ; \frac{3}{2} ; c t^{2}\right)}{16 c^{2}}-\frac{t_{2} F_{2}\left(\frac{1}{2} ; \frac{3}{2} ; c t^{2}\right)}{8 c}-\frac{p t_{2} F_{2}\left(\frac{1}{2} ; \frac{3}{2} ; c t^{2}\right)}{32 c^{2}}-\frac{t \rho_{2} F_{2}\left(\frac{1}{2} ; \frac{3}{2} ; c t^{2}\right)}{32 c^{2}}
\end{aligned}
$$

where the functions erfi $(x)$ and $\operatorname{erf}(x)$ denote the imaginary error function and the error function respectively, while the function ${ }_{p} F_{q}(a ; b ; z)$ stands for the generalized hypergeometric function. The Gauss-Bonnet invariant in this case is equal to,

$$
\mathcal{G}=-96 c^{2} t^{2}\left(2 c+4 c^{2} t^{2}\right)
$$

which can be solved with respect to $t$, and we have the following positive solution,

$$
t=\sqrt{-\frac{1}{4 c}+\frac{\sqrt{24 c^{6}-c^{4} G}}{8 \sqrt{6} c^{4}}}
$$

Having this at hand, and by substituting to Eq. (31), we easily obtain the function $f_{\mathcal{G}}(\mathcal{G})$. Then by integrating with respect to $\mathcal{G}$, we can get the resulting $f_{\mathcal{G}}(\mathcal{G})$ function, but we omit the resulting expression, since it's functional form is too large and complicated. Note however that all the integrals can be evaluated analytically, as the reader may convince himself.

Before closing, we study here a final example, for which we assume that $g_{0}=0$, in which case the function $g(t)$ is equal to,

$$
g(t)=-\frac{a^{3}(t)}{4 \dot{a}^{2}(t)} \int_{0}^{t} a^{-1}\left(t_{1}\right)\left(\frac{\ddot{a}\left(t_{1}\right)}{a\left(t_{1}\right)}-\frac{\dot{a}\left(t_{1}\right)}{a^{2}\left(t_{1}\right)}\right) d t_{1}-\frac{\tilde{C} a^{3}(t)}{8 \dot{a}^{2}(t)} \int_{0}^{t} a^{-4}\left(t_{1}\right) d t_{1} .
$$

Consider a cosmological evolution with scale factor $a(t)=(1+\alpha t)^{\gamma}$. The case $\alpha<0, \gamma<0$ corresponds to the Big Rip singularity [24], which occurs at $t_{s}=1 / \alpha$. The accelerated expansion corresponds to $\alpha>0, \gamma>1$. The corresponding solution for $g(t)$ reads,

$$
g(t)=-\frac{1}{4 \gamma^{2} \alpha^{2}}\left((1+\alpha t)^{2+\gamma}-(1+\alpha t)^{2}\right)-\frac{\tilde{C}}{8 \gamma^{2} \alpha^{3}(4 \gamma-1)}\left((1+\alpha t)^{2+\gamma}-(1+\alpha t)^{3(1-\gamma)}\right), \quad \gamma \neq 1 / 4 .
$$

The corresponding Gauss-Bonnet invariant $\mathcal{G}$ is,

$$
\mathcal{G}=24 \frac{\gamma^{3} \alpha^{4}(1-\gamma)}{(1+\alpha t)^{4}}
$$

so the resulting expression for the $f(\mathcal{G})$ gravity reads,

$$
f(\mathcal{G})=A \mathcal{G}^{1 / 4}+B \mathcal{G}^{(1-\gamma) / 4}+D \mathcal{G}^{3 \gamma / 4} .
$$




\section{IV. $f(\mathcal{G})$ GRAVITY IN FORMULATION WITH LAGRANGE MULTIPLIER}

In this section we consider the formulation of $f(\mathcal{G})$ modified gravity by using a Lagrange multiplier, and the impact of this multiplier to the cosmological evolution of various models. One can impose a condition on the scalar field by adding to the standard action for $f(\mathcal{G})$ gravity the following term,

$$
\lambda\left(g^{\mu \nu} \partial_{\mu} \phi \partial_{\nu} \phi+U(\phi)\right)
$$

where $\lambda$ is the Lagrange multiplier and $U(\phi)$ is a function of the scalar field, in principle arbitrarily chosen. We also include in the Jordan frame action of the $f(\mathcal{G})$ gravity, the action of a scalar field (canonical or phantom) $\phi$ with arbitrarily chosen scalar potential $V(\phi)$, which is of the form:

$$
S_{\phi}=\int d^{4} x \sqrt{-g}\left(-\epsilon g^{\mu \nu} \partial_{\mu} \phi \partial_{\nu} \phi-V(\phi)\right) .
$$

The cases $\epsilon= \pm 1$ describe canonical and phantom scalar field respectively. For $\epsilon=0$ the conformal symmetry of the system is respected and the corresponding gravity with Lagrange multiplier is equivalent to the mimetic $f(\mathcal{G})$ gravity considered above (for $U=1$ and $V=0$ ). (The case with non-zero $V$ maybe called as extended mimetic theory). The full Jordan frame action of the $f(\mathcal{G})$ gravity with Lagrange multiplier and in the presence of the scalar field and other matter fluids, is the following,

$$
S=\int d^{4} x \sqrt{-g}\left(\frac{R}{2}+f(\mathcal{G})-\epsilon g^{\mu \nu} \partial_{\mu} \phi \partial_{\nu} \phi-V(\phi)+\lambda\left(g^{\mu \nu} \partial_{\mu} \phi \partial_{\nu} \phi+U(\phi)\right)+\mathcal{L}_{m}\right)
$$

By varying this action with respect to the metric $g_{\mu \nu}$, we obtain the following two equations of motion:

$$
\begin{gathered}
3 H^{2}+24 H^{3} \frac{d f_{\mathcal{G}}(\mathcal{G})}{d t}+f(\mathcal{G})-f_{\mathcal{G}}(\mathcal{G}) \mathcal{G}=\rho+\epsilon \dot{\phi}^{2}+V(\phi)-\lambda\left(\dot{\phi}^{2}+U(\phi)\right), \\
-2 \dot{H}-3 H^{2}-8 H^{2} \frac{d^{2} f_{\mathcal{G}}(\mathcal{G})}{d t^{2}}-16 H\left(\dot{H}+H^{2}\right) \frac{d f_{\mathcal{G}}(\mathcal{G})}{d t}+f_{\mathcal{G}}(\mathcal{G}) \mathcal{G}-f(\mathcal{G})=p+\epsilon \dot{\phi}^{2}-V(\phi)-\lambda\left(\dot{\phi}^{2}-U(\phi)\right) .
\end{gathered}
$$

where we assumed a flat FRW metric. Also, by varying action (37), with respect to the Lagrange multiplier, provides us with the following constraint for the scalar field:

$$
\dot{\phi}^{2}-U(\phi)=0
$$

Finally for the scalar field we have the equation of motion:

$$
2 \partial_{t}((\lambda-\epsilon) \dot{\phi})+6 H(\lambda-\epsilon) \dot{\phi}-V^{\prime}(\phi)+\lambda U^{\prime}(\phi)=0 .
$$

For a Universe dominated by collisionless matter $(p=0)$, the scalar potential $V(t)$ and the Lagrange multiplier function $\lambda(t)$, as function of the cosmic time read,

$$
\begin{gathered}
V(t)=2 \dot{H}+3 H^{2}+\epsilon \dot{\phi}^{2}+8 H^{2} \frac{d^{2} f_{\mathcal{G}}(\mathcal{G})}{d t^{2}}+16 H\left(\dot{H}+H^{2}\right) \frac{d f_{\mathcal{G}}(\mathcal{G})}{d t}-f_{\mathcal{G}}(\mathcal{G}) \mathcal{G}+f(\mathcal{G}), \\
\lambda(t)=\dot{\phi}^{-2}\left(\frac{\rho}{2}+\dot{H}+4 H\left(2 \dot{H}-H^{2}\right) \frac{d f_{\mathcal{G}}(\mathcal{G})}{d t}+4 H^{2} \frac{d^{2} f_{\mathcal{G}}(\mathcal{G})}{d t^{2}}\right)+\epsilon
\end{gathered}
$$

Equation (41) follows easily by combining Eqs. (38) and (39). Therefore, for a given $f(\mathcal{G})$ one can specify the evolution of the scale factor (or the corresponding Hubble parameter) and obtain the scalar field potential which is responsible for this evolution. In addition, the inverse process is easy to be realized, that is, for a given scalar potential and for an arbitrarily given cosmological evolution, it is possible to reconstruct the corresponding $f(\mathcal{G})$ gravity. In the following sections, we exemplify the two aforementioned reconstruction methods. 


\section{A. Some Examples for $f(\mathcal{G})=A \mathcal{G}^{2}$ gravity and $\phi=\sqrt{U_{0}} t$}

The simplest choice for the function $f(\mathcal{G})$ is the following,

$$
f(\mathcal{G})=A \mathcal{G}^{2} .
$$

In this section, we shall assume that the scalar field $\phi$ is related to the cosmic time $t$ as, $\phi=\sqrt{U_{0}} t$, which means that $U(\phi)=U_{0}=$ const. For the bounce case with scale factor given in Eq. (29) and Hubble rate given in Eq. (30), the corresponding potential of the scalar field, provided by Eq. (42), takes the following form,

$$
V(t)=A \alpha^{5} U_{0}^{-1} \phi^{2} \sum_{n=0}^{3} C_{n}\left(\alpha U_{0}^{-1} \phi^{2}\right)^{n}+V_{0},
$$

where the parameter $V_{0}$, which is equal to,

$$
V_{0}=3(2 c)^{2} U_{0}^{-1} \phi^{2}+4 c+\epsilon U_{0}
$$

stands for the potential of the scalar field in the case of General Relativity (which can be achieved if $A=0$ ), and $C_{n}$ are numerical coefficients. For completeness, we provide the full expression for the scalar potential in the Appendix. The corresponding Lagrange multiplier function $\lambda(t)$, which is given in Eq. (43), is equal to,

$$
\lambda(\phi)=-\frac{36864 A c^{5}}{U_{0}}+\epsilon+\frac{2 c}{\phi^{2}}+\frac{\rho}{\phi^{2}}-\frac{221184 A c^{6} \phi^{2}}{U_{0}^{2}}+\frac{98304 A c^{7} \phi^{4}}{U_{0}^{3}}
$$

In the same way, we may easily find the scalar potential and the auxiliary function $\lambda(\phi)$, for the case of the superbounce with scale factor and Hubble rate given in Eqs. (22) and (23) respectively. The corresponding scalar potential is,

$$
\begin{aligned}
& V(\phi)=\frac{12288 A\left(-12+44 c^{2}-75 c^{4}+28 c^{6}\right) U_{0}^{4}}{c^{16}\left(-t_{s} \sqrt{U_{0}}+\phi\right)^{8}} \\
& +\frac{c^{12} \sqrt{U_{0}}\left(-t_{s} \sqrt{U_{0}}+\phi\right)^{6}\left(c^{4} t_{s}^{2} U_{0} \epsilon+c^{4} \epsilon \phi^{2}-2 \sqrt{U_{0}}\left(-6+2 c^{2}+c^{4} t_{s} \epsilon \phi\right)\right)}{c^{16}\left(-t_{s} \sqrt{U_{0}}+\phi\right)^{8}}
\end{aligned}
$$

while the Lagrange multiplier function $\lambda(\phi)$ equals to,

$$
\begin{aligned}
& \lambda(\phi)=\frac{24576 A\left(-4-12 c^{2}+7 c^{4}\right) U_{0}^{4}}{c^{14} \phi^{2}\left(-t_{s} \sqrt{U_{0}}+\phi\right)^{8}} \\
& +\frac{c^{12}\left(-t_{s} \sqrt{U_{0}}+\phi\right)^{6}\left(-2 c^{2} t_{s} \sqrt{U_{0}} \phi\left(\rho+\epsilon \phi^{2}\right)+c^{2} \phi^{2}\left(\rho+\epsilon \phi^{2}\right)+U_{0}\left(-2+c^{2} t_{s}^{2}\left(\rho+\epsilon \phi^{2}\right)\right)\right)}{c^{14} \phi^{2}\left(-t_{s} \sqrt{U_{0}}+\phi\right)^{8}}
\end{aligned}
$$

Another example that produces Little Rip cosmology, is described by the following Hubble rate,

$$
H(t)=H_{0} \exp (\alpha t), \quad \alpha>0 .
$$

with the corresponding scalar potential being of the following form,

$$
\begin{gathered}
V(\phi)=A H_{0}^{5} \exp (5 \gamma \phi) \sum_{n=0}^{3} C_{n} \alpha^{3-n} H_{0}^{n} \exp (n \gamma \phi)+V_{0}, \\
V_{0}=3 H_{0}^{2} \exp (2 \gamma \phi)+2 H_{0} \alpha \exp (\gamma \phi)+\epsilon U, \quad \gamma=\alpha U^{-1 / 2} .
\end{gathered}
$$

In order to better understand what is the difference between the Lagrange multiplier mimetic matter with a scalar potential, which was studied in Ref. [11, and Lagrange multiplier $f(\mathcal{G})$ gravity, we shall study in detail some examples that were presented in [11]. It is worth recalling some features of Lagrange multiplier mimetic matter, with scalar 
potential, and for a detailed presentation, the reader is referred to [11]. The action of the mimetic matter with scalar potential and with Lagrange multiplier, is given below [25],

$$
\left.S=\int \mathrm{d} x^{4}\left[-\frac{1}{2} R+\lambda\left(g^{\mu \nu} \partial_{\mu} \phi \partial_{\nu} \phi\right)-1\right)-V(\phi)+L_{m}\right]
$$

with $L_{m}$ denoting as usual the matter Lagrangian. In the context of the theory developed in [1], the Lagrange multiplier is equal to,

$$
\lambda=\frac{1}{2}(G-T-4 V(\phi)),
$$

with $G$ and $T$ standing for the trace of the Einstein tensor and for the trace of the energy momentum tensor respectively. The form of the Lagrange multiplier appearing in Eq. (511) is obviously different from the Lagrange multiplier appearing in Eq. (43), but one can easily verify that if we take $U(\phi)=1$ and also $f(\mathcal{G})=0$, these become identical. We now investigate the new features that the $f(\mathcal{G})$ modification brings along. We choose again the $f(\mathcal{G})$ gravity to be of the form given in Eq. (44) and also we assume that $\phi=\sqrt{U_{0}} t$. We shall start our comparison analysis, by studying the inflaton case presented in [11], where it was shown that a cosmological evolution with scale factor equal to,

$$
a(t)=e^{-\sqrt{\frac{\alpha}{12}} t^{2}}
$$

with $\alpha$ a constant parameter. The potential that generates this kind of evolution, always in the context of Ref. [11], is equal to,

$$
V(\phi)=\frac{\alpha \phi^{2}}{e^{\phi}+1}
$$

In contrast, in the case of the Lagrange multiplier $f(\mathcal{G})$ modified gravity, the potential that generates the cosmological evolution described by Eq. (52), is equal to,

$$
V(\phi)=-\frac{2 \sqrt{\alpha}}{\sqrt{3}}+\sqrt{U_{0}} \epsilon+\frac{\alpha \phi^{2}}{U_{0}}+\frac{256 A \alpha^{5 / 2} \phi^{2}}{\sqrt{3} U_{0}}-\frac{1088 A \alpha^{3} \phi^{4}}{3 U_{0}^{2}}+\frac{1408 A \alpha^{7 / 2} \phi^{6}}{9 \sqrt{3} U_{0}^{3}}-\frac{64 A \alpha^{4} \phi^{8}}{9 U_{0}^{4}},
$$

which is clearly much different than the one in Eq. (53). In addition, the corresponding Lagrange multiplier function $\lambda(\phi)$ is equal to,

$$
\lambda(\phi)=\epsilon-\frac{\sqrt{\alpha}}{\sqrt{3} \phi^{2}}+\frac{\rho}{\phi^{2}}+\frac{128 A \alpha^{5 / 2}\left(9 \sqrt{3} U_{0}^{2}-27 U_{0} \sqrt{\alpha} \phi^{2}-2 \sqrt{3} \alpha \phi^{4}\right)}{27 U_{0}^{3}}
$$

which is obviously different from the corresponding one in ordinary Lagrange multiplier mimetic matter theory given in Eq. (51). It is obvious that within the context of Lagrange multiplier $f(\mathcal{G})$ modified theory of gravity, there are many ways of generating various cosmological scenarios, since there is much freedom in choosing the potential $U(\phi)$ and also the function $f(\mathcal{G})$. In this way, almost any cosmological scenario can be generated, by suitably choosing the two aforementioned functions. Before closing this section, we shall study another example presented in [11], which describes mimetic matter as quintessence. The scale factor of the corresponding cosmological evolution, is equal to [11],

$$
a(t)=t^{\frac{2}{3(1+w)}}
$$

which actually describes the behavior of mimetic matter when the Universe is dominated by some other matter fluid present, with equation of state $p=w \rho$. The potential that generates the cosmological evolution (56), is equal to [11],

$$
V(\phi) \simeq \frac{\alpha}{t^{2}}
$$

where $\alpha$ is some arbitrary constant. In the case of Lagrange multiplier $f(\mathcal{G})$ gravity, the potential that can generate the cosmological evolution of Eq. (56), is equal to,

$$
V(\phi)=\frac{U_{0}^{4}\left(4096 A(1+3 w)(67+w(149+84 w))-\frac{972 w(1+w)^{6} \phi^{6}}{U_{0}^{3}}+\frac{729(1+w)^{8} \epsilon \phi^{8}}{U_{0}^{7 / 2}}\right)}{729(1+w)^{8} \phi^{8}},
$$


and the corresponding Lagrange multiplier function $\lambda(\phi)$, is equal to,

$$
\lambda(\phi)=\epsilon+\frac{8192 A U_{0}^{4}(1+3 w)(23+21 w)}{729(1+w)^{7} \phi^{10}}-\frac{2 U_{0}}{3(1+w) \phi^{4}}+\frac{\rho}{\phi^{2}} .
$$

which are clearly different from the ones given in Eqs. (57) and (51).

As we already mentioned, in the context of Lagrange multiplier $f(\mathcal{G})$ modified gravity, there is much freedom in reconstructing the scalar potential for the scalar field, since the function $U(\phi)$ and the function $f(\mathcal{G})$ itself can be arbitrarily chosen. Let us consider as a final example the case for which the function that relates the scalar field $\phi$ to the cosmic time $t$ is of the following form, $\phi=\sqrt{U_{0}} \ln t$, so the function $U(\phi)$ is of the following form,

$$
U(\phi)=U_{0} \exp \left(-2 U_{0}^{-1 / 2} \phi\right)
$$

Assuming that,

$$
H_{0}=\alpha t
$$

and also that the function $f(\mathcal{G})$ is again $f(\mathcal{G})=A \mathcal{G}^{2}$, the corresponding scalar field potential $V(\phi)$, is of the form,

$$
V(\phi)=A \alpha^{5} \exp \left(2 U_{0}^{-1 / 2} \phi\right) \sum_{n=0}^{3} C_{n} \alpha^{n} \exp \left(n U_{0}^{-1 / 2} \phi\right)+V_{0},
$$

where $V_{0}$ in this case stands for,

$$
V_{0}=2 \alpha+3 \alpha^{2} \exp \left(2 U_{0}^{-1 / 2} \phi\right)+\epsilon U_{0} \exp \left(-2 U_{0}^{-1 / 2} \phi\right) .
$$

In the same way, more elaborated universe evolution unifying inflation, dark energy and dark matter maybe presented.

\section{B. Reconstruction of $f(\mathcal{G})$ gravity given the Lagrange multiplier function and the scale factor}

In this section we consider the inverse reconstruction method, which enables us to find the Lagrange multiplier $f(\mathcal{G})$ modified gravity, given the scale factor of the cosmological evolution and the Lagrange multiplier function. Let us explicitly demonstrate how this method works. We start off with Eq. (43), which can be rewritten as:

$$
(\lambda(t)-\epsilon) \dot{\phi}^{2}-\frac{\rho}{2}-\dot{H}-4 a(t) \frac{d}{d t}\left(\frac{H^{2}}{a(t)} \frac{d f_{\mathcal{G}}}{d t}\right)=0 .
$$

Solving this equation with respect to $f_{\mathcal{G}}(t)$ we have:

$$
f_{\mathcal{G}}(t)=\frac{1}{4} \int d t_{1} \frac{a\left(t_{1}\right)}{H^{2}\left(t_{1}\right)} \int^{t_{1}} \frac{d t_{2}}{a\left(t_{2}\right)}\left(\left(\lambda\left(t_{2}\right)-\epsilon\right) \dot{\phi}^{2}\left(t_{2}\right)-\dot{H}\left(t_{2}\right)-\frac{\rho_{0}}{2 a^{3}\left(t_{2}\right)}\right) .
$$

Note that for $\lambda=1$, Eq. (63) in fact coincides with (35) (with $C=0$ ) for $g(t)=d f_{\mathcal{G}} / d t$, as was expected. The potential of scalar field as a function of time is cast as follows,

$$
\begin{aligned}
V(t)=(2 \lambda(t)-\epsilon) \dot{\phi}^{2}(t)+3 H^{2}(t)-\rho(t) & +6 a(t) H(t) \int \frac{d t_{1}}{a\left(t_{1}\right)}\left(\left(\lambda\left(t_{1}\right)-\epsilon\right) \dot{\phi}^{2}\left(t_{1}\right)-\dot{H}\left(t_{1}\right)-\frac{\rho_{0}}{2 a^{3}\left(t_{1}\right)}\right) \\
& -f_{\mathcal{G}}(t) \mathcal{G}(t)+f(t) .
\end{aligned}
$$

We assume that the function that relates the scalar field $\phi$ and the cosmic time $t$ is denoted as $t=y(\phi)$. We also write the scale factor $a(t)$ in the following form,

$$
a(t)=\exp (h(t))
$$

Then, the Hubble parameter is simply written in terms of $h(t)$, as $H(t)=\dot{h}(t)$. Considering for simplicity the case that no matter fluids are present, that is $\rho=0$, the potential of the scalar field given in Eq. (64), becomes,

$$
V(\phi)=3 \dot{h}^{2}(y(\phi))-\frac{\epsilon}{y^{\prime 2}(\phi)}-6 \mathrm{e}^{h(y(\phi)} \dot{h}(y(\phi)) \int d \phi_{1} y^{\prime}\left(\phi_{1}\right) \mathrm{e}^{-h\left(y\left(\phi_{1}\right)\right)}\left(\frac{\epsilon}{y^{\prime 2}\left(\phi_{1}\right)}+\ddot{h}\left(y\left(\phi_{1}\right)\right)\right)+
$$




$$
\begin{gathered}
+\frac{2 \lambda(\phi)}{y^{\prime 2}(\phi)}+6 \mathrm{e}^{h(y(\phi))} \dot{h}(y(\phi)) \int d \phi_{1} \mathrm{e}^{-h\left(y\left(\phi_{1}\right)\right)}\left(\frac{\lambda\left(\phi_{1}\right)}{y^{\prime}\left(\phi_{1}\right)}\right)- \\
-f_{\mathcal{G}}(\phi) \mathcal{G}(y(\phi))+\int d \phi_{1} f_{\mathcal{G}}\left(\phi_{1}\right) y^{\prime}\left(\phi_{1}\right) \mathcal{G}_{y}\left(y\left(\phi_{1}\right)\right)
\end{gathered}
$$

and therefore, the function $f_{\mathcal{G}}$ as a function of the scalar field is equal to,

$$
\begin{gathered}
f_{\mathcal{G}}(\phi)=-\frac{1}{4} \int d \phi_{1} \dot{h}^{-2}\left(y\left(\phi_{1}\right)\right) \mathrm{e}^{h\left(y\left(\phi_{1}\right)\right)} y^{\prime}\left(\phi_{1}\right) \int^{\phi_{1}} d \phi_{2} y^{\prime}\left(\phi_{2}\right) \mathrm{e}^{-h\left(y\left(\phi_{2}\right)\right)}\left(\frac{\epsilon}{y^{\prime 2}\left(\phi_{2}\right)}+\ddot{h}\left(y\left(\phi_{2}\right)\right)\right)+ \\
+\frac{1}{4} \int d \phi_{1} \dot{h}^{-2}\left(y\left(\phi_{1}\right)\right) \mathrm{e}^{h\left(y\left(\phi_{1}\right)\right)} y^{\prime}\left(\phi_{1}\right) \int^{\phi_{1}} d \phi_{2} \mathrm{e}^{-h\left(y\left(\phi_{2}\right)\right)}\left(\frac{\lambda\left(\phi_{2}\right)}{y^{\prime}\left(\phi_{2}\right)}\right) .
\end{gathered}
$$

Then, for a given cosmological evolution, in terms of some specific scale factor, by choosing the arbitrary Lagrange multiplier function $\lambda(\phi)$, we can easily obtain the corresponding $f(\mathcal{G})$ gravity, and also finally find the resulting form of the potential $V(\phi)$, which we used earlier for the derivation of the $f(\mathcal{G})$ gravity.

Let us exemplify how this inverse reconstruction method works, by using some illustrative examples. For simplicity, we shall assume that the field $\phi$ is related to the cosmic time as, $\phi=\sqrt{U_{0}} t$, so we define the function $y(\phi)$ to be the following,

$$
y(\phi)=U_{0}^{-1 / 2} \phi
$$

which we extensively use in the following considerations. Of course, one can in principle choose a different function, but this will only perplex the equations, without changing the qualitative feature of our reconstruction method.

We start off with the superbounce, with scale factor and Hubble rate appearing in Eqs. (22) and (23) respectively. The function $h(t)$ is in this case equal to,

$$
h(t)=\ln \left(-t+t_{s}\right)^{\frac{2}{c^{2}}}
$$

In addition, we take the function $\lambda(t)$, to be equal to,

$$
\lambda(t)=c t
$$

Then, by combining Eqs. (67), (68), (69) and (70), we obtain the function $f_{\mathcal{G}}(\phi)$, which is equal to,

$$
\begin{aligned}
& f_{\mathcal{G}}(\phi)=\frac{c^{7}\left(-\frac{c^{2} t_{s}^{4} \phi}{\sqrt{U_{0}}}+\frac{\left(1+c^{2}\right) t_{s}^{3} \phi^{2}}{\sqrt{U_{0}}}-\frac{2 t_{s}^{2} \phi^{3}}{\sqrt{U_{0}}}-\frac{\left(-3+c^{2}\right) t_{s} \phi^{4}}{2 \sqrt{U_{0}}}+\frac{\left(-2+c^{2}\right) \phi^{5}}{5 \sqrt{U_{0}}}\right)}{32\left(2-3 c^{2}+c^{4}\right) U_{0}} \\
& -\frac{c^{4} \phi\left(-2 t_{s}+\phi\right)\left(2 c^{2}\left(2+c^{2}\right) t_{s}^{2} \epsilon-2 c^{2}\left(2+c^{2}\right) t_{s} \epsilon \phi+U_{0}\left(-8+\frac{c^{4} \epsilon \phi^{2}}{U_{0}}+2 c^{2}\left(2+\frac{\epsilon \phi^{2}}{U_{0}}\right)\right)\right)}{64\left(-4+c^{4}\right) U_{0}}
\end{aligned}
$$

Then, the $f(\mathcal{G})$ gravity easily follows, by using the expression for the Gauss-Bonnet invariant, which for the case of the superbounce is given in Eq. (25), by integrating with respect to $\mathcal{G}$. The final expression is quite large and for simplicity reasons we give it in the Appendix. In the same way, by using Eqs. (66), (68), (69) and (700), we obtain 
the potential of the scalar field, which is,

$$
\begin{aligned}
& V(\phi)=-\frac{\epsilon}{U_{0}}+\frac{12}{c^{4}\left(t_{s}-\phi\right)^{2}}+\frac{2 c \phi}{U_{0}^{3 / 2}}-\frac{6 c\left(2 t_{s} \phi-2 \phi^{2}+c^{2}\left(-t_{s}^{2}+\phi^{2}\right)\right)}{\left(2-3 c^{2}+c^{4}\right) U_{0}^{3 / 2}\left(t_{s}-\phi\right)} \\
& -\frac{12\left(c^{2}\left(2+c^{2}\right) t_{s}^{2} \epsilon-2 c^{2}\left(2+c^{2}\right) t_{s} \epsilon \phi+U_{0}\left(-4+\frac{c^{4} \epsilon \phi^{2}}{U_{0}}+2 c^{2}\left(1+\frac{\epsilon \phi^{2}}{U_{0}}\right)\right)\right)}{c^{2}\left(-4+c^{4}\right) U_{0}\left(t_{s}-\phi\right)^{2}} \\
& +\frac{96\left(\frac{4}{c^{4}\left(t_{s}-\phi\right)^{2}}-\frac{2}{c^{2}\left(t_{s}-\phi\right)^{2}}\right)\left(c^{7}\left(-\frac{c^{2} t_{s}^{4} \phi}{\sqrt{U_{0}}}+\frac{\left(1+c^{2}\right) t_{s}^{3} \phi^{2}}{\sqrt{U_{0}}}-\frac{2 t_{s}^{2} \phi^{3}}{\sqrt{U_{0}}}-\frac{\left(-3+c^{2}\right) t_{s} \phi^{4}}{2 \sqrt{U_{0}}}+\frac{\left(-2+c^{2}\right) \phi^{5}}{5 \sqrt{U_{0}}}\right)\right)}{c^{4}\left(t_{s}-\phi\right)^{2} 32\left(2-3 c^{2}+c^{4}\right) U_{0}} \\
& -\frac{96\left(\frac{4}{c^{4}\left(t_{s}-\phi\right)^{2}}-\frac{2}{c^{2}\left(t_{s}-\phi\right)^{2}}\right)\left(c^{4} \phi\left(-2 t_{s}+\phi\right)\left(2 c^{2}\left(2+c^{2}\right) t_{s}^{2} \epsilon-2 c^{2}\left(2+c^{2}\right) t_{s} \epsilon \phi+U_{0}\left(-8+\frac{c^{4} \epsilon \phi^{2}}{U_{0}}+2 c^{2}\left(2+\frac{\epsilon \phi^{2}}{U_{0}}\right)\right)\right)\right)}{c^{4}\left(t_{s}-\phi\right)^{2} 64\left(-4+c^{4}\right) U_{0}} \\
& -\frac{3\left(-2+c^{2}\right)\left(t_{s}^{2}\left(-2 c^{3} t_{s}^{3}+5 c^{5} t_{s}^{3}+3 c^{7} t_{s}^{3}-40 U_{0}^{3 / 2}-5 c^{6} t_{s}^{2} \sqrt{U_{0}} \epsilon-5 c^{4} \sqrt{U_{0}}\left(4 U_{0}+t_{s}^{2} \epsilon\right)+10 c^{2} \sqrt{U_{0}}\left(6 U_{0}+t_{s}^{2} \epsilon\right)\right)\right)}{5 c^{4} U_{0}^{3 / 2}\left(4-4 c^{2}-c^{4}+c^{6}\right)\left(t_{s}-\phi\right)^{4}} \\
& +\frac{+\frac{40 U_{0}^{3 / 2}}{\left(2+c^{2}\right)\left(t_{s}-\phi\right)^{2}}+\frac{8 c^{3} \phi}{-1+c^{2}}+\frac{20 c^{2}\left(c t_{s}-\sqrt{\sigma_{0}} \epsilon\right) \ln \left[-t_{s}+\phi\right]}{-2+c^{2}}}{5 c^{4} U_{0}^{3 / 2}}
\end{aligned}
$$

In the same way, we can easily obtain the $f(\mathcal{G})$ gravity for the case of the bounce with scale factor (29). Using the same conventions as in the case of the superbounce, the resulting expression for the $f(\mathcal{G})$ gravity is,

$$
\begin{aligned}
& f(\mathcal{G})=-\frac{1}{4 U_{0}^{2}} c \sqrt{-\frac{4}{c}+\frac{\sqrt{16 c^{6}-\frac{2 c^{4} G}{3}}}{c^{4}}} \\
& \times\left(8+\frac{\sqrt{16 c^{6}-\frac{2 c^{4} G}{3}}}{c^{3}}+\frac{3}{16} c\left(-\frac{4}{c}+\frac{\sqrt{16 c^{6}-\frac{2 c^{4} G}{3}}}{c^{4}}\right)^{3 / 2} \sqrt{\pi} U_{0}\left(2 c U_{0}+\epsilon\right)\right) \\
& \times G_{34}^{22}\left(\begin{array}{c}
-1,0,1 \\
0,0,-2,-\frac{1}{2}
\end{array} \mid \frac{12 c^{3} U_{0}-\sqrt{144 c^{6}-6 c^{4} G} U_{0}}{48 c^{3}}\right) \\
& +\frac{1}{4} \sqrt{\frac{-36 c^{3} \pi+3 \sqrt{144 c^{6}-6 c^{4} G} \pi}{c^{4}}} U_{0}\left(2 c U_{0}+\epsilon\right) G_{34}^{22}\left(\begin{array}{c}
0,0,1 \\
0,0,-1,-\frac{1}{2}
\end{array} \mid \frac{12 c^{3} U_{0}-\sqrt{144 c^{6}-6 c^{4} G} U_{0}}{48 c^{3}}\right)
\end{aligned}
$$

with $G_{p q}^{m n}$, the Meijer $G$-function. The resulting expression for the scalar potential is too complicated, so we provide the details for it in the Appendix.

As a final example, we shall consider a non-monotonic evolution for the Hubble rate which has the following form,

$$
H(t)=\frac{2}{t}+\frac{2}{t_{f}-t}
$$

Notice that a Big Rip singularity occurs at $t=t_{f}$. It easily follows that,

$$
\begin{gathered}
h(\phi)=\ln \left(\frac{\phi}{U_{0}}\right)-\ln \left(\frac{\left(\phi_{f}-\phi\right)^{2}}{U_{0}}\right), \\
\dot{h}(\phi)=\frac{2 \sqrt{U_{0}}}{\phi}+\frac{2 \sqrt{U_{0}}}{\phi_{f}-\phi}, \quad \ddot{h}(\phi)=-\frac{2 U_{0}}{\phi^{2}}+\frac{2 U_{0}}{\left(\phi_{f}-\phi\right)^{2}}, \quad \phi_{f}=\sqrt{U_{0}} t_{f} .
\end{gathered}
$$

Without getting into too much detail, the function $f_{\mathcal{G}}(\phi)$ is equal to,

$$
f_{\mathcal{G}}(\phi)=-\frac{1}{16 U_{0} \phi_{f}^{2}}\left(\frac{\epsilon \phi^{6}}{6}+C_{1} \phi^{5}+\frac{2 \epsilon \phi_{f}}{25} \phi^{5}-\frac{2 \epsilon \phi_{f}}{5} \phi^{5} \ln \phi-\frac{\epsilon \phi_{f}^{2}}{4} \phi^{4}-\frac{2 \phi_{f}}{3} \phi^{3}+\frac{\phi_{f}^{2}}{2} \phi^{2}+C_{2}\right)+
$$




$$
+\frac{1}{4} \int d \phi_{1} \frac{\dot{h}^{-2}\left(y\left(\phi_{1}\right)\right) \mathrm{e}^{h\left(y\left(\phi_{1}\right)\right)}}{y^{\prime 2}\left(\phi_{1}\right)} \int^{\phi_{1}} d \phi_{2} \mathrm{e}^{-h\left(y\left(\phi_{2}\right)\right)}\left(\frac{\lambda\left(\phi_{2}\right)}{y^{\prime}\left(\phi_{2}\right)}\right)
$$

while the corresponding scalar field potential is equal to,

$$
\begin{gathered}
V(\phi)=\frac{U_{0}}{\left(\phi_{f}-\phi\right)^{3}}\left(4 \phi_{f}^{3} \phi^{-2}+12 \phi_{f}^{2} \phi^{-1}+\epsilon\left(11 \phi_{f}^{3}+3 \phi_{f}^{2} \phi-15 \phi_{f} \phi^{2}+\phi^{3}+24 \phi_{f}^{2} \phi \ln \phi\right)-60 C_{1} \phi_{f} \phi\right)+ \\
+\frac{2 \lambda(\phi)}{y^{\prime 2}(\phi)}+6 \mathrm{e}^{h(y(\phi))} \dot{h}(y(\phi)) \int d \phi_{1} \mathrm{e}^{-h\left(y\left(\phi_{1}\right)\right)}\left(\frac{\lambda\left(\phi_{1}\right)}{y^{\prime}\left(\phi_{1}\right)}\right)- \\
-\int d \phi_{1} \frac{f_{\mathcal{G}}\left(\phi_{1}\right)}{d \phi_{1}} \mathcal{G}\left(y\left(\phi_{1}\right)\right) .
\end{gathered}
$$

Notice that the last integral can be evaluated analytically, but we omit the resulting expression for the sake of brevity. In conclusion, with this inverse reconstruction method, if the Lagrange multiplier function $\lambda(\phi)$ and the scale factor $a(t)$ are specified, the corresponding Lagrange multiplier $f(\mathcal{G})$ gravity follows, as we explicitly demonstrated in the previous examples. Of course, it is to be understood that if the integrals cannot be evaluated analytically, further assumptions should be taken into account, but the general theoretical framework is the same.

\section{DISCUSSION}

It is worth discussing certain issues related to the mimetic $f(\mathcal{G})$ gravity models we studied in this paper. In particular one possible question that comes to mind when dealing with this kind of theories is the following: all the models we constructed in the present study involve an $f(\mathcal{G})$ function, with $\mathcal{G}$ being the Gauss-Bonnet term which in the usual case is harmless, since it can be integrated out topologically. Now the question is if the theory constructed by using a function of the Gauss-Bonnet invariant, contains any extra degrees of freedom or not. In our case however, note that the $f(\mathcal{G})$ gravity is considered here as an effective string-inspired gravity theory. Due to non-linear functional dependence on $\mathrm{G}$, the function $f(\mathcal{G})$ is not a topological invariant anymore. The introduction of the $f(\mathcal{G})$ term, introduces to the theory an effective scalar degree of freedom, as in many cases the model maybe rewritten as one containing a string-inspired $V(\phi) \mathcal{G}$ term. In addition, in the context of the mimetic $f(\mathcal{G})$ gravity, there is an extra scalar degree of freedom, that of the scalar field $\phi$, which accounts for the dark matter content of the theory. Now we come to another important issue that we need to briefly discuss. As we demonstrated in this paper, the mimetic $f(\mathcal{G})$ cosmological scenario can serve as a theoretical framework that at the same time can describe inflation, dark energy and even dark matter. Although it is quite appealing to have a geometric description of the cosmic history of our Universe, an important question rises, with regards to the inflationary particle content of the theory. In particular, for inflation, the most important and successful achievement was to predict a nearly scale invariant power spectrum which is verified by CMB experiments in the past decade. However, this achievement is based on the fundamental assumption that inflation is realized by a slow-rolling scalar field in the early universe. Without such a scalar field, how can the CMB spectrum be realized in modified gravity models?

This question is very interesting and we shall try to briefly address this, so let us first consider the standard mimetic gravity, presented in [10, 11], where the authors considered the standard Einstein-Hilbert mimetic extension. As it was shown in [11], the metric perturbation is related to the higher derivative of the scalar field's linear perturbation $\dot{\delta} \phi$, and in effect, the resulting picture is that, the perturbations behave as dust, with the speed of sound being zero, even for mimetic matter. Therefore, the quantum fluctuations of mimetic matter are ill-defined, and therefore the large scale structure cannot be explained by the minimal Einstein-Hilbert mimetic matter theory. An extra scalar field is required to play the role of the curvaton or explicitly modify the mimetic dark matter model, in order for the large scale structure being consistently described. Therefore, the mimetic $f(\mathcal{G})$ could potentially provide such an extension, by offering extra degrees of freedom to the theory. The corresponding extra scalar degree of freedom we discussed earlier, may play such a role in the resulting theory. In [11], the authors modified by hand the minimally extended mimetic Einstein-Hilbert Lagrangian, thus being able to produce a red-tilted spectral index. It would be therefore quite interesting to investigate whether this extra scalar term can originate by an $f(\mathcal{G})$ theory or from any other mimetic extension of modified gravity. However, this task exceeds the purposes of this paper and we hope to address such issues in the future. 


\section{CONCLUSIONS}

In this paper we considered the generalization of ordinary Jordan frame $f(\mathcal{G})$ gravity, to mimetic $f(\mathcal{G})$ gravity. In this new theory, in principle it is possible to resolve two important cosmological problems, firstly to obtain the Universe's accelerated expansion without the need to introduce extra fields (inflaton, dark energy scalar) and secondly to provide an answer to the problem of dark matter. Specifically, within the context of mimetic $f(\mathcal{G})$ gravity, the cosmological equations of motion are almost the same with these that result in ordinary Jordan frame $f(\mathcal{G})$ gravity, with the only difference being that the contribution of mimetic matter appears in the mimetic $f(\mathcal{G})$ gravity case. After having provided the formalism of mimetic $f(\mathcal{G})$ gravity, we were able to realize various cosmological evolutions with mimetic $f(\mathcal{G})$ gravity, and highlighted the difference between ordinary and mimetic $f(\mathcal{G})$ gravity. In principle, in the case of mimetic $f(\mathcal{G})$ gravity, there is much more freedom in providing the $f(\mathcal{G})$ gravity that can realize a certain cosmological evolution. This can be one of the attributes of the theory, since a quite large number of cosmological evolutions can be successfully realized in the context of mimetic $f(\mathcal{G})$ gravity. We also compared the resulting mimetic $f(\mathcal{G})$ gravities, to standard mimetic gravity models that exist in the literature.

We also modified the ordinary Jordan frame $f(\mathcal{G})$ gravity, to include a Lagrange multiplier term. After describing in detail how the cosmological equations are modified within this new formalism, we provided two quite general reconstruction techniques, which in principle can be useful for the realization of various cosmological models. Particularly, it is possible in the context of Lagrange multiplier $f(\mathcal{G})$ gravity, given the cosmological evolution and the $f(\mathcal{G})$ gravity, to find the scalar field potential $V(\phi)$ and the Lagrange multiplier function $\lambda(t)$. In addition, the inverse procedure is possible, that is, given the cosmological evolution and the Lagrange multiplier function $\lambda(t)$, it is possible to reconstruct the corresponding $f(\mathcal{G})$ gravity. We supported our theoretical considerations by using some illustrative examples, for which we applied both reconstruction methods.

As a general remark, we must note that in the context of both mimetic $f(\mathcal{G})$ gravity and Lagrange multiplier $f(\mathcal{G})$ gravity, there exist a large class of $f(\mathcal{G})$ gravities that can realize a specific cosmological evolution. We believe that this feature is an attribute of the theory, since it is possible, by suitably choosing the parameters and functions, to get analytic results. This was not always possible in ordinary Jordan frame $f(\mathcal{G})$ gravity.

An issue we did not address in this paper is the realization of singular cosmology, in the context of mimetic $f(\mathcal{G})$ gravity or Lagrange multiplier $f(\mathcal{G})$ gravity. By singular cosmology we mean the appearance of finite time singularities [26] (with the Big Rip 24] being the most elaborated one) in the cosmological evolution. Among all the finite time singularities, the most interesting ones are the non-crushing types singularities, for example, Type II [27] or the mildest among them, the Type IV [26, 28]. Since these singularities can be consistently incorporated in scalar-tensor theories [28], it would be interesting to realize such singular cosmological evolution from mimetic $f(\mathcal{G})$ and from Lagrange multiplier $f(\mathcal{G})$, and compare the results to ordinary $f(\mathcal{G})$ gravity. Special emphasis for this task, should be given near the Type IV singularity of course. Work is in progress towards this direction and we hope to report on this issue soon.

Finally, let us discuss another issue having to do with the possible observational distinction between the various modified gravity theories. Indeed, these theories can in principle be very degenerate with regards to their observational prediction so the fundamental question that comes to mind is, whether there is any possible way of distinguishing these theories at a pragmatic level. One good idea is to these these modified gravity models by using weak lensing data, owing to the fact that the trajectories of light may differ, depending on different geometric terms. For some relevant works on this issue see for example [29]. In addition, the growth index may provide some useful insights on that respect, see for example [30].

\section{Acknowledgments}

This work is supported in part by project 14-02-31100 (RFBR, Russia) and project 2058 (MES, Russia) (AVA), by MINECO (Spain), projects FIS2010-15640 and FIS2013-44881 (SDO).

\section{Appendix: Resulting expressions for $f(\mathcal{G})$ gravities and for scalar potentials}

Here we quote the complicated expressions we mentioned in the main text of this paper. The full expression for the potential appearing in Eq. (45) is equal to,

$$
V(\phi)=4 c+12 c^{2} t^{2}-73728 A c^{5} t^{2}-626688 A c^{6} t^{4}-540672 A c^{7} t^{6}-147456 A c^{8} t^{8}+\sqrt{U_{0}} \epsilon
$$


In addition, by integrating Eq. (71), with respect to $\mathcal{G}$, we obtain the $f(\mathcal{G})$ function, which is equal to,

$$
\begin{aligned}
& f(\phi(\mathcal{G}))=-\frac{1}{c^{4} U_{0}} 12\left(-2+c^{2}\right)\left(\frac{2 c^{3} U_{0}^{3 / 2} \phi(\mathcal{G})}{5\left(-1+c^{2}\right)}-\frac{1}{20\left(4-4 c^{2}-c^{4}+c^{6}\right)\left(-t_{s}+\frac{\phi(\mathcal{G})}{\sqrt{U_{0}}}\right)^{4}}\right. \\
& \times t_{s}^{2}\left(2 c^{3} t_{s}^{3} \sqrt{U_{0}}\left(10-20 \sqrt{U_{0}}+15 U_{0}-4 U_{0}^{3 / 2}\right)+5 c^{5} t_{s}^{3}\left(-4+6 \sqrt{U_{0}}\right.\right. \\
& \left.-4 U_{0}+U_{0}^{3 / 2}\right)-40\left(-2 U_{0}^{3 / 2}+U_{0}^{2}\right)+c^{7} t_{s}^{3}\left(-10+10 \sqrt{U_{0}}-5 U_{0}^{3 / 2}+2 U_{0}^{2}\right) \\
& -5 c^{6} t_{s}^{2} \sqrt{U_{0}}\left(-4+6 \sqrt{U_{0}}-4 U_{0}+U_{0}^{3 / 2}\right) \epsilon-5 c^{4}\left(-2 \sqrt{U_{0}}+U_{0}\right)\left(2 t_{s}^{2} \epsilon-2 t_{s}^{2} \sqrt{U_{0}} \epsilon+U_{0}\left(4+t_{s}^{2} \epsilon\right)\right) \\
& \left.+10 c^{2}\left(-2 \sqrt{U_{0}}+U_{0}\right)\left(2 t_{s}^{2} \epsilon-2 t_{s}^{2} \sqrt{U_{0}} \epsilon+U_{0}\left(6+t_{s}^{2} \epsilon\right)\right)\right) \\
& -\frac{1}{3\left(4-4 c^{2}-c^{4}+c^{6}\right)\left(-t_{s}+\frac{\phi(\mathcal{G})}{\sqrt{U_{0}}}\right)^{3}} 2 t_{s}\left(-1+\sqrt{U_{0}}\right)\left(2 c^{5} t_{s}^{3}\left(-1+\sqrt{U_{0}}\right)^{2}+c^{7} t_{s}^{3}\left(-1+\sqrt{U_{0}}\right)^{2}\left(1+\sqrt{U_{0}}\right)\right. \\
& -4 c^{3} t_{s}^{3}\left(-1+\sqrt{U_{0}}\right)^{2} \sqrt{U_{0}}-8 U_{0}^{3 / 2}-2 c^{6} t_{s}^{2}\left(-1+\sqrt{U_{0}}\right)^{2} \sqrt{U_{0}} \epsilon \\
& \left.-2 c^{4} \sqrt{U_{0}}\left(t_{s}^{2} \epsilon-2 t_{s}^{2} \sqrt{U_{0}} \epsilon+U_{0}\left(2+t_{s}^{2} \epsilon\right)\right)+4 c^{2} \sqrt{U_{0}}\left(t_{s}^{2} \epsilon-2 t_{s}^{2} \sqrt{U_{0}} \epsilon+U_{0}\left(3+t_{s}^{2} \epsilon\right)\right)\right) \\
& +\frac{1}{\left(4-4 c^{2}-c^{4}+c^{6}\right)\left(-t_{s}+\frac{\phi(\mathcal{G})}{\sqrt{U_{0}}}\right)^{2}}\left(-3 c^{5} t_{s}^{3}\left(-1+\sqrt{U_{0}}\right)^{2} \sqrt{U_{0}}+2 c^{3} t_{s}^{3}\left(-1+\sqrt{U_{0}}\right)^{2}\right. \\
& \times\left(-1+4 \sqrt{U_{0}}\right) \sqrt{U_{0}}+4 U_{0}^{2}-c^{7} t_{s}^{3} \sqrt{U_{0}}\left(1-3 U_{0}+2 U_{0}^{3 / 2}\right)+3 c^{6} t_{s}^{2}\left(-1+\sqrt{U_{0}}\right)^{2} U_{0} \epsilon \\
& \left.-6 c^{2} U_{0}\left(U_{0}+t_{s}^{2} \epsilon-2 t_{s}^{2} \sqrt{U_{0}} \epsilon+t_{s}^{2} U_{0} \epsilon\right)+c^{4} U_{0}\left(3 t_{s}^{2} \epsilon-6 t_{s}^{2} \sqrt{U_{0}} \epsilon+U_{0}\left(2+3 t_{s}^{2} \epsilon\right)\right)\right) \\
& -\frac{4 c^{2} t_{s}\left(-1+\sqrt{U_{0}}\right) U_{0}\left(c\left(t_{s}-2 t_{s} \sqrt{U_{0}}\right)+c^{3} t_{s} \sqrt{U_{0}}+\sqrt{U_{0}} \epsilon-c^{2} \sqrt{U_{0}} \epsilon\right)}{\left(2-3 c^{2}+c^{4}\right)\left(-t_{s}+\frac{\phi(\mathcal{G})}{\sqrt{U_{0}}}\right)} \\
& \left.+\frac{c^{2} U_{0}^{3 / 2}\left(c t_{s}\left(3-4 \sqrt{U_{0}}\right)+c^{3} t_{s}\left(-1+2 \sqrt{U_{0}}\right)+\sqrt{U_{0}} \epsilon-c^{2} \sqrt{U_{0}} \epsilon\right) \ln \left[-t_{s}+\frac{\phi(\mathcal{G})}{\sqrt{U_{0}}}\right]}{2-3 c^{2}+c^{4}}\right)
\end{aligned}
$$

where $\phi(\mathcal{G})$ stands for,

$$
\phi(\mathcal{G})=\frac{2 \sqrt{2} 3^{1 / 4} c^{4} \sqrt{G}\left(c^{8}\left(-2+c^{2}\right) G\right)^{1 / 4}+c^{8} G t_{s}}{c^{8} G\left(\sqrt{U_{0}}\right)^{-1}}
$$

or equivalently,

$$
\phi(\mathcal{G})=\frac{-2 \sqrt{2} 3^{1 / 4} c^{4} \sqrt{G}\left(c^{8}\left(-2+c^{2}\right) G\right)^{1 / 4}+c^{8} G t_{s}}{c^{8} G\left(\sqrt{U_{0}}\right)^{-1}}
$$

In conclusion, we have two different types of $f(\phi(\mathcal{G}))$ gravity, which can be found by substituting the two different forms of $\phi(\mathcal{G})$ to Eq. (77). Finally, the corresponding potential for the $f(\mathcal{G})$ gravity of Eq. (73), which corresponds 
to the bounce (29), is equal to,

$$
\begin{aligned}
& V(\phi)=-\frac{\epsilon}{U_{0}}-\frac{4 c \phi}{U_{0}^{3 / 2}}+12 c^{2} \phi^{2}-\frac{6 \sqrt{c} e^{c \phi^{2}} \sqrt{\pi}\left(2 c U_{0}+\epsilon\right) \phi \operatorname{erf}[\sqrt{c} \phi]}{U_{0}} \\
& +96 c^{2} \phi^{2}\left(2 c+4 c^{2} \phi^{2}\right)\left(\frac{1}{32 c^{2} U_{0}^{3 / 2} \phi}+\frac{\sqrt{\pi}\left(2 c U_{0}+\epsilon\right) G_{23}^{21}\left(\begin{array}{c}
0,1 \\
0,0,-\frac{1}{2}
\end{array} \mid-c \phi^{2}\right)}{64 c^{2} U_{0}}\right) \\
& \left.-\frac{c \phi\left(4\left(3+4 c \phi^{2}\right)+12 c \sqrt{\pi} \sqrt{U_{0}}\left(2 c U_{0}+\epsilon\right) \phi^{3} G_{34}^{22}\left(\begin{array}{c}
-1,0,1 \\
\left.0,0,-2,-\frac{1}{2} \mid-c \phi^{2}\right)
\end{array}\right)\right)}{U_{0}^{3 / 2}}\right) \\
& +\frac{c \phi\left(3 \sqrt{\pi} \sqrt{U_{0}}\left(2 c U_{0}+\epsilon\right) \phi G_{23}^{21}\left(\begin{array}{c}
0,1 \\
0,0,-\frac{1}{2}
\end{array} \mid-c \phi^{2}\right)\right)}{U_{0}^{3 / 2}}
\end{aligned}
$$

where $G_{p q}^{m n}$ is the Meijer G-function and $\operatorname{erf}(x)$ is the error function.

[1] P. A. R. Ade et al. [Planck Collaboration], arXiv:1502.02114 [astro-ph.CO]. ;

P. A. R. Ade et al. [Planck Collaboration], Astron. Astrophys. 571 (2014) A22 arXiv:1303.5082 [astro-ph.CO]].

[2] P. A. R. Ade et al. [BICEP2 Collaboration], Phys. Rev. Lett. 112 (2014) 24, 241101 [arXiv:1403.3985 [astro-ph.CO]].

[3] S. Capozziello, Int. J. Mod. Phys. D 11, 483 (2002).

[4] S.M. Carroll, V. Duvvuri, M. Trodden and M.S. Turner, Phys. Rev. D 70, 043528 (2004).

[5] A. G. Riess et al. [Supernova Search Team Collaboration], Astron. J. 116 (1998) 1009 astro-ph/9805201.

[6] S. Nojiri, S. D. Odintsov, Int.J.Geom.Meth.Mod.Phys. 11 (2014) 1460006 arXiv:1306.4426;

S. Nojiri, S. D. Odintsov, Int. J. Geom. Meth. Mod.Phys. 4 (2007) 115 hep-th/0601213;

S. Capozziello, V. Faraoni, Beyond Einstein Gravity, Springer, Berlin 2010;

S. Nojiri, S. D. Odintsov, Phys.Rept. 505 (2011) 59 arXiv:1011.0544;

S. Capozziello, M. De Laurentis, Phys.Rept. 509 (2011) 167 arXiv:1108.6266;

A. de la Cruz-Dombriz, D. Saez-Gomez, Entropy 14 (2012) 1717 arXiv:1207.2663.

[7] S. Nojiri and S. D. Odintsov, Phys. Rev. D 68 (2003) 123512 hep-th/0307288.

[8] W. Hu, I. Sawicki, Phys.Rev.D76 (2007) 064004 arXiv:0705.1158; ;

G. Cognola, E. Elizalde, S. Nojiri, S. D. Odintsov, L. Sebastiani and S. Zerbini, Phys. Rev. D 77 (2008) 046009 arXiv:0712.4017 [hep-th]];

S. Nojiri and S. D. Odintsov, Phys. Rev. D 77 (2008) 026007 arXiv:0710.1738 [hep-th]]

[9] S. Nojiri, S. D. Odintsov, Phys.Lett. B657 (2007) 238 arXiv:0707.1941;

S. Capozziello, S. Nojiri, S.D. Odintsov, A. Troisi, Phys.Lett. B639 (2006) 135 astro-ph/0604431;

S. Capozziello, V.F. Cardone, S. Carloni, A. Troisi, Int.J.Mod.Phys. D12 (2003) 1969 astro-ph/0307018;

A.S. Chudaykin, D.S. Gorbunov, A.A. Starobinsky, R.A. Burenin arXiv:1412.5239;

R. Myrzakulov, L. Sebastiani, S. Zerbini, Int.J.Mod.Phys. D22 (2013) 1330017 arXiv:1302.4646;

A. de la Cruz-Dombriz and A. Dobado, Phys. Rev. D 74 (2006) 087501 gr-qc/0607118;

S. Capozziello, M. De Laurentis and V. Faraoni, Open Astron. J. 3 (2010) 49 [arXiv:0909.4672 [gr-qc]];

G. J. Olmo, Int. J. Mod. Phys. D 20 (2011) 413 arXiv:1101.3864 [gr-qc]];

A. Capolupo, S. Capozziello, G. Vitiello, Int.J.Mod.Phys. A23 (2008) 4979 arXiv:0705.0319;

P. K.S. Dunsby, E. Elizalde, R. Goswami, S. Odintsov, D. S. Gomez, Phys.Rev. D82 (2010) 023519 arXiv:1005.2205;

S. Nojiri, S. D. Odintsov, D. Saez-Gomez, Phys.Lett. B681 (2009) 74 arXiv:0908.1269;

S. Capozziello, V. F. Cardone, A. Troisi, Phys.Rev. D71 (2005) 043503 astro-ph/0501426;

V. Faraoni, Phys.Rev. D74 (2006) 104017 astro-ph/0610734;

S. A. Appleby, R. A. Battye, Phys.Lett.B654 (2007) 7 arXiv:0705.3199;

S. A. Appleby, R. A. Battye, JCAP 0805 (2008) 019 arXiv:0803.1081;

V. Faraoni, Phys.Rev. D75 (2007) 067302 gr-qc/0703044

[10] A. H. Chamseddine and V. Mukhanov, JHEP 1311 (2013) 135 arXiv:1308.5410 [astro-ph.CO]].

[11] A. H. Chamseddine, V. Mukhanov and A. Vikman, JCAP 1406 (2014) 017 arXiv:1403.3961 [astro-ph.CO]].

[12] A. Golovnev, Phys. Lett. B 728 (2014) 39 arXiv:1310.2790 [gr-qc]];

N. Deruelle and J. Rua, arXiv:1407.0825 [gr-qc];

D. Momeni, A. Altaibayeva and R. Myrzakulov, arXiv:1407.5662 [gr-qc]; J. Matsumoto, S. D. Odintsov and S. V. Sushkov, Phys. Rev. D 91 (2015) 6, 064062 arXiv:1501.02149 [gr-qc]]; G. Leon and E. N. Saridakis, arXiv:1501.00488 [gr-qc]; 
R. Myrzakulov and L. Sebastiani, arXiv:1503.04293 [gr-qc]; D. Momeni, R. Myrzakulov and E. Gdekli, arXiv:1502.00977 $[\mathrm{gr}-\mathrm{qc}]$;

[13] S. Nojiri and S. D. Odintsov, Mod. Phys. Lett. A 29 (2014) 40, 1450211 arXiv:1408.3561 [hep-th]].

[14] S. Nojiri and S. D. Odintsov, Phys. Lett. B 631 (2005) 1 hep-th/0508049.

[15] S. Capozziello, A. N. Makarenko and S. D. Odintsov, Phys. Rev. D 87 (2013) 8, 084037 arXiv:1302.0093 [gr-qc]]; S. Capozziello, M. Francaviglia and A. N. Makarenko, Astrophys. Space Sci. 349 (2014) 603 [arXiv:1304.5440 [gr-qc]].

[16] M. Novello, S.E.Perez Bergliaffa, Phys.Rept. 463 (2008) 127 arXiv:0802.1634.

[17] Y. F. Cai, T. Qiu, Y. S. Piao, M. Li and X. Zhang, JHEP 0710 (2007) 071 [arXiv:0704.1090 [gr-qc]].

[18] N. Arkani-Hamed, Hsin-Chia Cheng, M. A. Luty, S. Mukohyama, JHEP 0405 (2004) 074 hep-th/0312099; A. Nicolis, R. Rattazzi, E. Trincherini, Phys.Rev. D79 (2009) 064036 arXiv:0811.2197; C. Deffayet, G. Esposito-Farese (Paris, Inst. Astrophys.), A. Vikman, Phys.Rev. D79 (2009) 084003 arXiv:0901.1314; J. Khoury, B. A. Ovrut, J. Stokes, JHEP 1208 (2012) 015 arXiv:1203.4562

[19] M. Koehn, Jean-Luc Lehners, B. A. Ovrut, Phys.Rev. D90 (2014) 025005 arXiv:1310.7577.

[20] Yi-Fu Cai, D. A. Easson, R. Brandenberger, JCAP 1208 (2012) 020 arXiv:1206.2382; J. Khoury, B. A. Ovrut, N. Seiberg, P. J. Steinhardt, N. Turok, Phys.Rev. D65 (2002) 086007 hep-th/0108187.

[21] S. D. Odintsov, V. K. Oikonomou and E. N. Saridakis, arXiv:1501.06591 [gr-qc].; V. K. Oikonomou, arXiv:1412.4343 [gr-qc].

[22] Yi-Fu Cai, Emmanuel N. Saridakis, Class. Quantum Grav. 28 (2011) 035010 arXiv:1007.3204

[23] S. Nojiri, S. D. Odintsov and M. Sami, Phys. Rev. D 74, 046004 (2006) ; D. Bazeia, B. Carneiro da Cunha, R. Menezes and A. Y. Petrov, Phys. Lett. B 649, 445 (2007). ; G. Cognola, E. Elizalde, S. Nojiri, S. D. Odintsov and S. Zerbini, Phys. Rev. D 73, 084007 (2006) ; G. Cognola, E. Elizalde, S. Nojiri, S. Odintsov and S. Zerbini, Phys. Rev. D 75, 086002 (2007) ; B. Li, J. D. Barrow and D. F. Mota, Phys. Rev. D 76, 044027 (2007) ; D. Bazeia, R. Menezes and A. Y. Petrov, Eur. Phys. J. C 58, 171 (2008) ; N. Goheer, R. Goswami, P. K. S. Dunsby and K. Ananda, Phys. Rev. D 79, 121301 (2009) ; N. Goheer, R. Goswami, P. K. S. Dunsby and K. Ananda Phys. Lett. B 679, 302 (2009) ; M. Mohseni, Phys. Lett. B 682, 89 (2009) ;

H. Mohseni Sadjadi, Europhys. Lett. 92, 50014 (2010) ;

M. Alimohammadi and A. Ghalee, Phys. Rev. D 79, 063006 (2009) ;

C. G. Boehmer and F. S. N. Lobo, Phys. Rev. D 79, 067504 (2009);

K. Uddin, J. E. Lidsey and R. Tavakol, Gen. Rel. Grav. 41, 2725 (2009) ;

S. Y. Zhou, E. J. Copeland and P. M. Saffin, JCAP 0907, 009 (2009) ;

M. M. Ivanov and A. V. Toporensky, Grav. Cosmol. 1843 (2012) ;

S. Nojiri, S. D. Odintsov, A. Toporensky and P. Tretyakov, Gen. Rel. Grav. 421997 (2010) ;

A. Kumar Sanyal, C. Rubano, E. Piedipalumbo, Gen.Rel.Grav. 43 2807, (2011) L. N. Granda, Mod. Phys. Lett. A 27, $1250018(2012)$;

L. N. Granda, Mod. Phys. Lett. A 28, 1350117 (2013) ;

E. Elizalde, R. Myrzakulov, V. V. Obukhov and D. Sez-Gmez, Class. Quant. Grav. 27, 095007 (2010) A. de la Cruz-Dombriz and D. Saez-Gomez Class. Quantum Grav. 29, 245014 (2012). S. Capozziello, M. De Laurentis and S. D. Odintsov, Mod. Phys. Lett. A 29 (2014) 30, 1450164 arXiv:1406.5652] [gr-qc]];

K. Bamba, Zong-Kuan Guo, N. Ohta, Prog.Theor.Phys. 118 (2007) 879 arXiv:0707.4334;

K. Bamba, S. D. Odintsov, L. Sebastiani, S. Zerbini, Eur.Phys.J. C67 (2010) 295 arXiv:0911.4390];

K. Bamba, A. N. Makarenko, A. N. Myagky, S. D. Odintsov, Phys.Lett. B732 (2014) 349 arXiv:1403.3242

[24] R. R. Caldwell, M. Kamionkowski and N. N. Weinberg, Phys. Rev. Lett. 91 (2003) 071301 astro-ph/0302506; B. McInnes, JHEP 0208 (2002) 029 arXiv:hep-th/0112066;

S. Nojiri and S. D. Odintsov, Phys. Lett. B 562, 147 (2003) arXiv:hep-th/0303117;

S. Nojiri and S. D. Odintsov, Phys. Rev. D 72 (2005) 023003 hep-th/0505215;

V. Gorini, A. Kamenshchik and U. Moschella, Phys. Rev. D 67 (2003) 063509 astro-ph/0209395;

E. Elizalde, S. Nojiri and S. D. Odintsov, Phys. Rev. D 70 (2004) 043539 hep-th/0405034. ;

V. Faraoni, Int. J. Mod. Phys. D 11, 471 (2002) arXiv:astro-ph/0110067;

P. Singh, M. Sami and N. Dadhich, Phys. Rev. D 68, 023522 (2003) arXiv:hep-th/0305110;

S. Nojiri and S. D. Odintsov, Phys. Rev. D 70 (2004) 103522 hep-th/0408170.;

C. Csaki, N. Kaloper and J. Terning, Annals Phys. 317, 410 (2005) arXiv:astro-ph/0409596];

P. X. Wu and H. W. Yu, Nucl. Phys. B 727, 355 (2005) arXiv:astro-ph/0407424];

M. Sami and A. Toporensky, Mod. Phys. Lett. A 19, 1509 (2004) arXiv:gr-qc/0312009];

H. Stefancic, Phys. Lett. B 586, 5 (2004) arXiv:astro-ph/0310904;

L. P. Chimento and R. Lazkoz, Phys. Rev. Lett. 91, 211301 (2003) arXiv:gr-qc/0307111;

Mod. Phys. Lett. A 19, 2479 (2004) arXiv:gr-qc/0405020;

J. G. Hao and X. Z. Li, Phys. Lett. B 606, 7 (2005) arXiv:astro-ph/0404154];

E. Babichev, V. Dokuchaev and Yu. Eroshenko, Class. Quant. Grav. 22, 143 (2005) arXiv:astro-ph/0407190;

X. F. Zhang, H. Li, Y. S. Piao and X. M. Zhang, Mod. Phys. Lett. A 21, 231 (2006) arXiv:astro-ph/0501652;

E. Elizalde, S. Nojiri, S. D. Odintsov and P. Wang, Phys. Rev. D 71, 103504 (2005) arXiv:hep-th/0502082;

M. P. Dabrowski and T. Stachowiak, Annals Phys. 321, 771 (2006) arXiv:hep-th/0411199;

F. S. N. Lobo, Phys. Rev. D 71, 084011 (2005) arXiv:gr-qc/0502099; 
R. G. Cai, H. S. Zhang and A. Wang, Commun. Theor. Phys. 44, 948 (2005) arXiv:hep-th/0505186;

I. Y. Aref'eva, A. S. Koshelev and S. Y. Vernov, Theor. Math. Phys. 148, 895 (2006) [Teor. Mat. Fiz. 148, 23 (2006)] arXiv:astro-ph/0412619;

Phys. Rev. D 72, 064017 (2005) arXiv:astro-ph/0507067;

H. Q. Lu, Z. G. Huang and W. Fang, arXiv:hep-th/0504038.

W. Godlowski and M. Szydlowski, Phys. Lett. B 623, 10 (2005) arXiv:astro-ph/0507322;

J. Sola and H. Stefancic, Phys. Lett. B 624, 147 (2005) arXiv:astro-ph/0505133;

B. Guberina, R. Horvat and H. Nikolic, Phys. Rev. D 72, 125011 (2005) arXiv:astro-ph/0507666;

M. P. Dabrowski, C. Kiefer and B. Sandhofer, Phys. Rev. D 74, 044022 (2006) arXiv:hep-th/0605229;

E. M. Barbaoza and N. A. Lemos, arXiv:gr-qc/0606084.

[25] E. A. Lim, I. Sawicki and A. Vikman, JCAP 1005 (2010) 012 arXiv:1003.5751 [astro-ph.CO]].

[26] S. Nojiri, S. D. Odintsov and S. Tsujikawa, Phys. Rev. D 71, 063004 (2005) arXiv:hep-th/0501025].

[27] J. D. Barrow, Class. Quant. Grav. 21 (2004) L79 gr-qc/0403084;

J. D. Barrow, Class. Quant. Grav. 21 (2004) 5619 gr-qc/0409062;

V. Sahni and Y. Shtanov, JCAP 0311, 014 (2003) arXiv:astro-ph/0202346];

S. Nojiri and S. D. Odintsov, Phys. Lett. B 595 (2004) 1 hep-th/0405078;

M. P. Dabrowski, Phys. Rev. D 71, 103505 (2005) arXiv:gr-qc/0410033;

L. Fernandez-Jambrina and R. Lazkoz, Phys. Rev. D 70, 121503 (2004) arXiv:gr-qc/0410124

[28] S. D. Odintsov and V. K. Oikonomou, arXiv:1504.01772 [gr-qc].;

S. Nojiri, S. D. Odintsov, V. K. Oikonomou and E. N. Saridakis, arXiv:1503.08443 [gr-qc].;

S. Nojiri, S. D. Odintsov and V. K. Oikonomou, arXiv:1502.07005 [gr-qc].

[29] F. Schmidt, Phys. Rev. D 78 (2008) 043002 [arXiv:0805.4812].

C. D. Leonard, T. Baker and P. G. Ferreira, Phys. Rev. D 91 (2015) 8, 083504 arXiv:1501.03509].

[30] S. A. Thomas, F. B. Abdalla and J. Weller, Mon. Not. Roy. Astron. Soc. 395 (2009) 197 arXiv:0810.4863. 